TITLE:

\title{
The Complexity of Selecting Maximal Solutions
}

\section{$\operatorname{AUTHOR}(\mathrm{S})$ :}

Chen, Zhi-Zhong; Toda, Seinosuke

\section{CITATION:}

Chen, Zhi-Zhong ... [et al]. The Complexity of Selecting Maximal Solutions. 数理解析研究所 講究録 1993, 833: 11-21

ISSUE DATE:

1993-04

URL:

http://hdl.handle.net/2433/83421

RIGHT: 


\title{
The Complexity of Selecting Maximal Solutions
}

\author{
Zhi-Zhong Chen \\ Department of Information Engineering, Mie University, Tsu-shi, Mie 514, Japan. \\ Seinosuke Toda \\ Department of Computer Science, University of Electro-Communications, \\ Chofu-shi, Tokyo 182, Japan.
}

\section{Introduction}

Intuitively, a maximization problem is to select a maximal solution for a given input according to some selection criterion. The maximal independent set problem (MIS) [5] and the minimal unsatisfiability problem (MinUnsat) [11] are two standard examples of such problems. Much work has been devoted to the study of maximization problems $[1,2,3,4,5,7,9,11,12]$. Most of the previous work has involved studying specific maximization problems and either finding an efficient algorithm (e.g., [5]) or proving the problem is hard to solve (e.g., [11]). An attractive alternative approach is to study maximization problems in a general framework and to prove general results.

In this paper, we formalize a maximization problem (MAXP) $Q$ as a pair $(D, R)$, where $D$ is the set of instances and $R: D \times\{0,1\}^{*} \rightarrow\{$ true, false $\}$ is the instancesolution relation. The objective in solving $Q$ is to select, given an instance $x \in D$, a maximal solution, i.e., a binary string $y$ such that $R(x, y)$ is true but changing one or more arbitrary 0 -bits of $y$ to 1 -bits will change the value of $R(x, y)$ to false. As an example, consider MIS in our framework. For it, $D$ is the set of all undirected graphs, and $R\left(G, b_{1} b_{2} \cdots b_{n}\right)$ is true if and only if $G$ has $n$ vertices (say, $\left.1,2, \cdots, n\right)$ and $\{i$ : $\left.b_{i}=1\right\}$ is an independent set in $G$. Our goal is to demonstrate what factors make $Q$ easy or hard to solve and how the factors influence the complexity of solving $Q$. We are able to find two such factors. One obvious factor is the complexity of $R$. This can be seen by comparing MIS with MinUnsat. The instance-solution relation of MIS is decidable in NC while that of MinUnsat is coNP-complete. Because of this gap, solving MinUnsat is much harder than solving MIS. In fact, MIS is solvable in NC [5,7] while solving MinUnsat is $D^{P}$-hard [11]. The other factor is whether $R$ is hereditary or not, where $R$ is said to be hereditary if and only if for every $x$ and $w$, whenever $R(x, w)$ is true, $R(x, w)$ remains true even one or more arbitrary 1-bits of $w$ are changed to 0 -bits. The instance-solution relation of MIS (also MinUnsat) is hereditary. In [9], Papadimitriou considered the following problem (MinModel): Given a CNF boolean formula $\phi$, find a satisfying truth assignment $\vec{a}$ to $\phi$ such that changing one or more arbitrary 1-bits of $\vec{a}$ to 0 -bits will make $\vec{a}$ no longer satisfy $\phi$. The instance-solution relation of MinModel is not hereditary but is decidable in NC. Unlike MIS, solving MinModel is obviously NP-hard.

In this paper, we restrict to consider only those MAXP's whose instance-solution relation is decidable in NP or coNP. We first consider upper bounds on the complexity of solving such MAXP's. Let $Q=(D, R)$ be a MAXP. The following give trivial upper bounds: (i) $Q$ is solvable in FP if $R$ is decidable in $\mathrm{P}$ and hereditary; (ii) $Q$ is solvable in NPMV //OptP $[O(\log n)]$ if $R$ is decidable in NP; (iii) $Q$ is solvable in FP NP $^{\text {if }} R$ is decidable in coNP and hereditary; (iv) $Q$ is solvable in $\mathrm{FP}_{2}^{\Sigma_{2}^{P}}$ if $R$ is decidable in coNP. 
Our main results concerning upper bounds are the following:

(v) Suppose $Q$ is a MAXP whose instance-solution relation is NP decidable. Let $e$ be an arbitrary polynomial. Then, there exist a function $F \in \mathrm{FP}_{\|}^{\mathrm{NP}}$ and a polynomial $p$ such that for every $x, \operatorname{Pr}[F(x, w)$ is a maximal solution of $x$ in $Q] \geq 1-2^{-e(|x|)}$, where $w \in\{0,1\}^{p(|x|)}$ is randomly chosen under uniform distribution.

(vi) Suppose $Q$ is a MAXP whose instance-solution relation is coNP decidable. Let $e$ be an arbitrary polynomial. Then, there exist a function $F \in \mathrm{FP}_{\|}^{\Sigma_{2}^{\mathrm{P}}}$ and a polynomial $p$ such that for every $x, \operatorname{Pr}[F(x, w)$ is a maximal solution of $x$ in $Q] \geq 1-2^{-e(|x|)}$, where $w \in\{0,1\}^{p(|x|)}$ is randomly chosen under uniform distribution.

(v) and (vi) are shown by extending the technique used in [3].

We then show that NPMV//OptP $[O(\log n)]$ is also a lower bound for solving those MAXP's whose instance-solution relation is decidable in NP or is decidable in $\mathrm{P}$ but not hereditary, and that $\mathrm{FP}_{\|}^{\mathrm{R}_{2}^{\mathrm{s}}}$ is also a lower bound for solving those MAXP's whose instancesolution relation is decidable in coNP but not hereditary. Combining the upper and lower bounds, we obtain characterizations of NPMV//OptP $[O(\log n)]$ and $\mathrm{FP}_{\|}^{\Sigma_{2}^{P}}$ via MAXP's. As an important consequence of the characterization of NPMV//OptP $[O(\log n)]$, we obtain the first natural complete problem for $\mathrm{NPMV} / / \mathrm{OptP}[O(\log n)]$. The problem (called $X$-MinModel) is defined as follows: Given a CNF boolean formula $\phi$ and a subset $X$ of the set of variables in $\phi$, find a satisfying truth assignment $\vec{a}$ to $\phi$ such that changing one or more arbitrary 1-bits of $\vec{a}$ corresponding to variables in $X$ to 0 -bits will make $\vec{a}$ no longer satisfy $\phi$. $X$-MinModel was first considered by Papadimitriou in [9], and was claimed without a precise proof to be $\Delta_{2}^{\mathrm{P}}$-complete there. However, Papadimitriou later withdrew his claim and thus left the complexity of $X$-MinModel open [10]. In [3], we proved that the complexity of $X$-MinModel is roughly captured by $\mathrm{FP}_{\|}^{\mathrm{NP}}$. Now, the results in this paper give, for the first time, the exact complexity of solving $X$-MinModel.

We also characterize complexity classes of sets via MAXP's. The following are shown:

(a) coNP is the class of all sets $L$ that can be expressed as $L=\{x: f(x)$ is a maximal solution of $x$ in $Q\}$ for some $f \in \mathrm{FP}$ and some MAXP $Q$ whose instance-solution relation is $\mathrm{P}$-decidable.

(b) $D^{\mathrm{P}}$ is the class of all sets $L$ that can be expressed as $L=\{x: f(x)$ is a maximal solution of $x$ in $Q\}$ for some $f \in \mathrm{FP}$ and some MAXP $Q$ whose instance-solution relation is NP-decidable.

(c) $\mathrm{D}^{\mathrm{P}}$ is the class of all sets $L$ that can be expressed as $L=\{x: f(x)$ is a maximal solution of $x$ in $Q\}$ for some $f \in \mathrm{FP}$ and some MAXP $Q$ whose instance-solution relation is coNP-decidable and hereditary.

(d) $\Pi_{2}^{\mathrm{P}}$ is the class of all sets $L$ that can be expressed as $L=\{x: f(x)$ is a maximal solution of $x$ in $Q\}$ for some $f \in \mathrm{FP}$ and some MAXP $Q$ whose instance-solution relation is coNP-decidable.

As consequences, we obtain several new natural problems that are $\leq_{m}^{\mathbf{P}}$-complete for coNP or $\mathrm{D}^{\mathrm{P}}$.

\section{Preliminaries}

We use $\Sigma=\{0,1\}$ as our alphabet. By a set, we mean a subset of $\Sigma^{*}$. Similarly, by a string, we mean a string in $\Sigma^{*}$. We denote by $|x|$ the length of a finite string $x$. The bits 
of a finite string with length $n$ are indexed from left to right as the 1st, 2 nd, $\cdots, n$th bits, respectively. For a finite string $x$, we usually identify $x$ with the set of all indices $i$ such that the $i$ th bit of $x$ is 1 . Thus we will often use some set-theoretical notations for finite strings. A finite string $x$ is smaller than another finite string $y$ if either $|x|<|y|$ or $|x|=|y|$ and $x \subset y$. A maximal string in a set $S$ of finite strings is a string in $S$ that is not smaller than any other string in $S$.

We assume a one-to-one pairing function from $\Sigma^{*} \times \Sigma^{*}$ to $\Sigma^{*}$ that is polynomial-time computable and polynomial-time invertible. For strings $x$ and $y$, we denote the output of the pairing function by $\langle x, y\rangle$; this notation is extended to denote any $k$-tuples for $k>2$ in a usual manner. W.l.o.g., we assume that $|\langle x, y\rangle|$ depends only on $|x|$ and $|y|$.

For any finite set $A,\|A\|$ denotes the number of strings in $A$. For a set $L, \bar{L}$ denotes its complement (i.e., $\Sigma^{*}-L$ ), and $\chi_{L}$ denotes the characteristic function of $L$. For a class $\mathbf{C}$ of sets, coC denotes the class of all sets whose complement is in $\mathbf{C}$. Let $\Sigma^{n}$ denote the set of all strings with length $n$. For two sets $L_{1}$ and $L_{2}, L_{1} \oplus L_{2}$ denotes the set $\left\{0 x: x \in L_{1}\right\} \cup\left\{1 y: y \in L_{2}\right\}$.

All functions considered here are ones from $\Sigma^{*}$ to $\Sigma^{*} \cup\{\#\}$. The symbol \# is assumed to be not in $\Sigma^{*}$. We consider both single-valued functions and multi-valued functions, but by a function we mean a (partial) single-valued function. For a multi-valued function $G, G(x)$ denotes the set of all possible values of $G$ at $x$. Thus, when $G(x)=\emptyset$, the multi-valued function $G$ is undefined at the argument $x$.

We assume that the reader is familiar with the basic concepts from the theory of computational complexity. Our computational models are variations of standard Turing machines. A machine is either an acceptor or a transducer, and may be deterministic or nondeterministic. An acceptor is denoted by $M$ or $M_{i}$ while a transducer is denoted by $T$ or $T_{i}$. A deterministic (resp., nondeterministic) Turing machine is abbreviated as DTM (resp., NTM). On a given input, a branch of a (nondeterministic) machine may halt by entering either a rejecting state or an accepting state. For simplicity, we say that a branch of a machine halts if the branch halts by entering an accepting state. Let $L(M)$ denote the set of all strings accepted by $M$. A transducer $T$ computes a string $y$ on input $x$ if some branch of $T$ on input $x$ halts with $y$ on the output tape. $T(x)$ denotes the set of all strings computed by $T$ on input $x$. A DTM $T$ computes a function $f$ if for all $x \in \Sigma^{*}, T(x)=\emptyset$ if $f(x)$ is undefined, and the unique element of $T(x)$ is $f(x)$ otherwise.

Classes in the first three levels of the polynomial-time hierarchy are denoted in the usual way: $\mathrm{P}, \mathrm{NP}, \operatorname{coNP}, \Sigma_{2}^{\mathrm{P}}, \Pi_{2}^{\mathrm{P}}=\operatorname{co} \Sigma_{2}^{\mathrm{P}}$. Let $\mathrm{D}^{\mathrm{P}}=\left\{L_{1} \cap L_{2}: L_{1} \in \mathrm{NP}\right.$ and $\left.L_{2} \in \operatorname{coNP}\right\}$.

FP denotes the class of all functions computed by polynomial-time bounded DTM's. Let $A$ be a set. $\mathrm{FP}^{A}$ denotes the class of all functions computed by polynomial-time bounded deterministic oracle Turing machines (DOTM) with oracle $A$. FP $A$ denotes the class of all functions $F$ for which there exists a polynomial-time bounded DOTM $T$ such that $T$, while computing $F(x)$ for a given $x$, prepares all its query strings before asking them to the oracle $A$. More precisely, a function $F$ is in $\mathrm{FP}_{\|}^{A}$ if there exist two functions $f$ and $g$ in FP such that for all strings $x, F(x)=g\left(x, \chi_{A}\left(y_{1}\right) \cdots \chi_{A}\left(y_{m}\right)\right)$, where $f(x)=\left\langle y_{1}, \cdots, y_{m}\right\rangle$. For a class $\mathbf{C}$ of sets, $\mathrm{FP}^{\mathbf{C}}=\cup_{\boldsymbol{A} \in \mathbf{C}} \mathrm{FP}^{\boldsymbol{A}}$ and $\mathrm{FP}_{\|}^{\mathbf{C}}=\cup_{\boldsymbol{A} \in \mathbf{C} \mathrm{FP}_{\|}^{\boldsymbol{A}}}$.

An NP metric Turing machine is a polynomial-time bounded NTM $T$ such that on every input, every branch of $T$ outputs a binary number and halts [6]. OptP $[O(\log n)]$ denotes the class of all (total) integer-valued functions $H$ for which there exist a polynomial $p$ and an NP metric Turing machine $T$ such that for every $x, H(x) \leq p(|x|)$ and 
$H(x)$ equals to the maximum number in $T(x)$. NPMV //OptP $[O(\log n)]$ denotes the class of all (partial) multi-valued functions $G$ for which there exist an NTM $T$ and a function $H \in \operatorname{OptP}[O(\log n)]$ such that for every $x, G(x)=T(\langle x, H(x)\rangle)$.

A maximization problem (MAXP) $Q$ is a pair $(D, R)$, where (i) $D$ is the set of instances and (ii) $R: D \times \Sigma^{*} \rightarrow\{$ true, false $\}$ is the instance-solution relation.

$R$ is said to be hereditary if for every $x \in D$ and every $w \in \Sigma^{*}$, whenever $R(x, w)$ is true, $R\left(x, w^{\prime}\right)$ is also true for every $w^{\prime}$ with $\left|w^{\prime}\right|=|w|$ and $w^{\prime} \subset w$. Let $x \in D$. A string $w$ is called a solution of $x$ if $R(x, w)$ is true. A maximal solution of $x$ is a maximal string in the set of all solutions of $x$. The objective in solving $Q$ is to compute, given an instance $x \in D$, a maximal solution of $x$.

Each MAXP $Q=(D, R)$ considered in this paper is required to satisfy the following: (1) $D$ is P-decidable (i.e., decidable in polynomial time), (2) there is a polynomial $p$ such that for every $x \in D$ and every string $w$, whenever $R(x, w)$ is true, $|w| \leq p(|x|)$, and (3) $R$ is NP-decidable or coNP-decidable.

Definition 2.1 A function $F$ solves $Q$ if for every $x \in D$, (a) $F(x)$ is undefined if $x$ has no solution in $Q$ and (b) $F(x)$ is a maximal solution of $x$ in $Q$ otherwise. A multi-valued function $G$ solves $Q$ if for every $x \in D$, (a) $G(x)=\emptyset$ if $x$ has no solution in $Q$ and (b) $G(x)$ is nonempty and each element of $G(x)$ is a maximal solution of $x$ in $Q$ otherwise. $Q$ is solvable in a class $\mathbf{H}$ of (single-valued or multi-valued) functions if some $H \in \mathbf{H}$ solves $Q$.

Definition 2.2 Let $F$ be a function, and let $G$ be a multi-valued function. Then, $F$ (resp., $G$ ) is reducible to $Q$ if there exist two functions $f, g$ in FP such that for every $x$, $f(x) \in D$ and $g(x, w)=F(x)$ (resp., $g(x, w) \in G(x)$ ) for every maximal solution $w$ of $f(x)$ in $Q . Q$ is hard for a class $\mathbf{H}$ of (single-valued or multi-valued) functions if every $H \in \mathbf{H}$ is reducible to $Q . Q$ is complete for a class $\mathbf{H}$ of (single-valued or multi-valued) functions if $Q$ is solvable in and hard for $\mathbf{H} . Q$ is hard for a class $\mathbf{C}$ of sets if $Q$ is hard for the class $\left\{\chi_{L}: L \in \mathbf{C}\right\}$.

Definition 2.3 The set $L_{Q}=\{\langle x, w\rangle: w$ is a maximal solution of $x$ in $Q\}$ is called the decision problem associated with $Q$.

\section{Upper bounds}

In this section, we show upper bounds on the complexity of solving MAXP's. The following proposition shows trivial upper bounds.

Proposition 3.1 Let $Q=(D, R)$ be a MAXP.

(1) If $R$ is hereditary and P-decidable, then $Q$ is solvable in FP.

(2) If $R$ is NP-decidable, then $Q$ is solvable in NPMV//OptP $[O(\log n)]$.

(3) If $R$ is hereditary and coNP-decidable, then $Q$ is solvable in FP ${ }^{\mathrm{NP}}$.

(4) If $R$ is coNP-decidable, then $Q$ is solvable in $\mathrm{FP}^{\Sigma_{2}^{\mathrm{P}}}$.

We next proceed to show two other non-trivial upper bounds. To do this, we need several definitions and a known result.

Definition 3.1 Let $\mathbf{F}$ be a class of functions. Then we define a class RP.F of 
multi-valued functions as follows: A multi-valued function $G$ is in RP.F if for every polynomial $e$, there exist a function $F \in \mathbf{F}$ and a polynomial $p$ such that for every string $x$, (a) $F(x, w)$ is undefined for all $w \in\{0,1\}^{p(|x|)}$ if $G(x)$ is undefined and (b) $\operatorname{Pr}[F(x, w) \in G(x) \cup\{\#\}]=1$ and $\operatorname{Pr}[F(x, w) \in G(x)] \geq 1-2^{-e(|x|)}$ otherwise, where $w$ is a random string chosen from $\{0,1\}^{p(|x|)}$. Intuitively speaking, $G$ is in RP.F if for every string $x$, we can randomly pick up an element of $G(x)$ using a function in $\mathbf{F}$.

Notation: For $k \geq 1,[1, k]$ denotes the set of all integers $i$ with $1 \leq i \leq k$.

Definition 3.2 Let $S$ be a finite set and let $k$ be a positive integer. A weight function over $S$ is a function from the elements of $S$ to positive integers. A $k$-weight function over $S$ is a weight function $f$ over $S$ such that for each $s \in S, f(s)$ is in $[1, k]$. A random $k$-weight function over $S$ is a $k$-weight function $f$ over $S$ such that for each $s \in S, f(s)$ is chosen uniformly and independently from $[1, k]$. The weight of a subset $S^{\prime}$ of $S$ under a weight function $f$ is $\Sigma_{\boldsymbol{s} \in S^{\prime}} f(s)$. Note that for every $k$-weight function over $S$, the weight of each subset of $S$ under $f$ is no more than $k\|S\|$ and that the empty set $\emptyset$ is the unique subset of $S$ with weight 0 .

Lemma 3.1 [8]. Let $\mathbf{S}$ be a nonempty family of subsets of a finite set $S$. Then, for any random $k$-weight function $f$ over $S$ with $k \geq 2\|S\|, \operatorname{Pr}$ [There is a unique maximum weight set in $\mathbf{S}$ under $f] \geq \frac{1}{2}$.

Now we are ready to show the two non-trivial upper bounds. The idea used in the proof is a generalization of the one used in [3].

Theorem 3.1 Let $Q=(D, R)$ be a MAXP.

(1) If $R$ is NP-decidable, then $Q$ is solvable in RP.FP $\|$.

(2) If $R$ is coNP-decidable, then $Q$ is solvable in RP.FP $\|_{2}^{\Sigma_{2}^{\mathrm{P}}}$.

Proof. We only show a proof for (2). (1) can be shown in a similar manner.

(2) We first explain the idea behind the proof. Let $p_{Q}$ be a polynomial such that for all $x \in D$, the length of each solution of $x$ is no more than $p_{Q}(|x|)$. Let $x$ be an instance of $Q$. Then, we consider $\mathbf{S}$, the family of all solutions of $x$ with maximum length. To find a maximal solution for $x$, we first get a random $2 p_{Q}(|x|)$-weight function $f$ over $\left[1, p_{Q}(|x|)\right]$. Then, by Lemma 3.1 , with probability at least $\frac{1}{2}$, there is a unique solution in $\mathbf{S}$ of maximum weight. To find this unique solution of maximum weight, it suffices to ask only one round of parallel queries to a $\Sigma_{2}^{P}$ oracle set. Since the weight assigned to each element of $\left[1, p_{Q}(|x|)\right]$ is positive, all maximum weight solutions are maximal solutions (but not necessarily solutions of maximum 1-bits). In order to get the high probability of success, we may perform several copies of this computation in parallel.

We now proceed to give the precise proof. Let $p_{Q}$ be a polynomial that bounds the lengths of solutions of $x$ from above. For convenience, let $n_{x}=p_{Q}(|x|)$ for all $x \in D$. Then we define five sets as follows:

$L_{R}=\{x: x$ has a solution $\}$,

$B_{1}=\left\{\langle x, i\rangle: 0 \leq i \leq n_{x}\right.$ and $x$ has a solution of length $\left.i\right\}$,

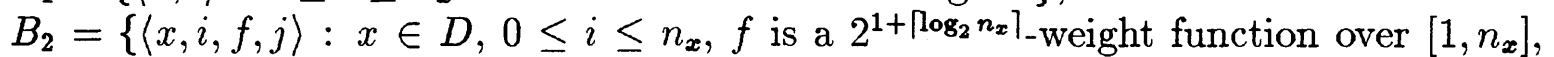
$0 \leq j \leq i 2^{\left.1+\log _{2} n_{x}\right\rceil}$, and $x$ has a solution $u$ such that $|u|=i$ and $j$ is the weight of $u$ under $f\}$,

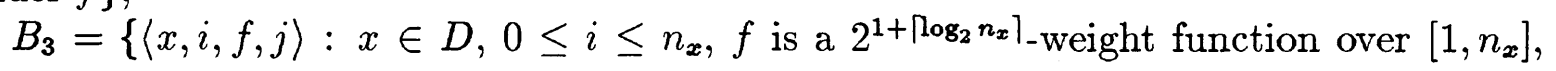


$0 \leq j \leq i 2^{1+\left\lceil\log _{2} n_{x}\right\rceil}$, and $x$ has two or more solutions $u$ such that $|u|=i$ and $j$ is the weight of $u$ under $f\}$, and

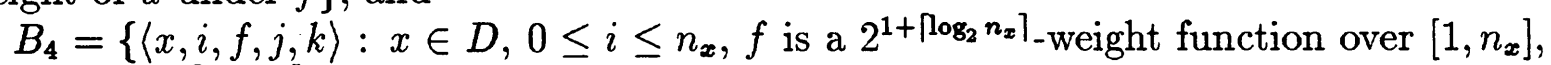
$0 \leq j \leq i 2^{1+\left\lceil\log _{2} n_{x}\right\rceil}, 1 \leq k \leq i$, and $x$ has a solution $u$ such that $|u|=i, j$ is the weight of $u$ under $f$, and the $k$ th bit of $u$ is 1 \}.

Obviously, $L_{R}, B_{1}, B_{2}, B_{3}$, and $B_{4}$ are in $\Sigma_{2}^{\mathrm{P}}$. Let $\left.B=\left(\left(\left(L_{R} \oplus B_{1}\right) \oplus B_{2}\right) \oplus B_{3}\right) \oplus B_{4}\right)$. Then, $B \in \Sigma_{2}^{\mathrm{P}}$.

Let $e$ be an arbitrary polynomial. We define a polynomial $p$ as follows: $p(i)=e(i)$. $\left(p_{Q}^{2}(i)+p_{Q}(i)\right)$. Below, we define a DOTM $T$ which uses $B$ as an oracle set. Given an input $\langle x, w\rangle$ with $x \in D$ and $w \in\{0,1\}^{p(|x|)}, T$ operates as follows:

Step 1: $T$ checks whether $x$ has a solution by asking a query to $L_{R}$. If $x$ has no solution, then $T$ halts by entering a rejecting state.

Step 2: $T$ finds $n_{1}$, the length of the longest solutions of $x$. This is done by asking the queries $\langle x, 0\rangle,\langle x, 1\rangle, \cdots,\left\langle x, n_{x}\right\rangle$ to the oracle set $B_{1}$.

Step 3: $T$ computes $n_{2}=2^{\left.1+\log _{2} n_{x}\right\rceil}$ and constructs, from $w, n_{2}$-weight functions $f_{1}$, $f_{2}, \cdots, f_{e(|x|)}$ over the set $\left[1, n_{x}\right]$ as follows:

Step 3.1: $T$ first computes $e(|x|)$ strings $w_{1}, \cdots, w_{\boldsymbol{e}(|x|)}$ from $w$ such that $\left|w_{1}\right|=\cdots=$ $\left|w_{e(|x|) \mid}\right|=n_{x} \log _{2} n_{2}$ and the string $w_{1} w_{2} \cdots w_{e(|x|)}$ is a prefix of $w$ (the remaining part of $w$ is ignored), and then for each $1 \leq k \leq e(|x|)$, it partitions $w_{k}$ into $n_{x}$ substrings $w_{k, 1}$, $\cdots, w_{k, n_{x}}$ each of length $\log _{2} n_{2}$. (Note: $T$ can do this because $n_{x}^{2}+n_{x} \geq n_{x} \log _{2} n_{2}$.)

Step 3.2: For each $1 \leq k \leq e(|x|)$ and each $l$ in $\left[1, n_{x}\right], T$ sets $f_{k}(l)=d_{k, l}+1$, where $d_{k, l}$ is the integer whose binary representation is $w_{k, l}$.

Step 4: For each $1 \leq k \leq e(|x|), T$ computes the maximum number $m_{k}$ with $\left\langle x, n_{1}, f_{k}, m_{k}\right\rangle \in B_{2}$. This is done by asking the queries $\left\langle x, i, f_{k}, j\right\rangle$ with $0 \leq i \leq n_{x}$, $1 \leq k \leq e(|x|)$, and $0 \leq j \leq i n_{2}$ to the oracle set $B_{2}$. (Note: In this step, $T$ asks the queries of the form $\left\langle x, i, f_{k}, j\right\rangle$ for all possible values of $i, k$, and $j$ because the machine needs to prepare all queries independently of each other.)

Step 5: For $1 \leq k \leq e(|x|)$ and $1 \leq l \leq n_{1}, T$ computes $a_{k, l}=\chi_{B_{4}}\left(\left\langle x, n_{1}, f_{k}, m_{k}, l\right\rangle\right)$. This is done by asking the queries $\left\langle x, i, f_{k}, j, l\right\rangle$ with $0 \leq i \leq n_{x}, 1 \leq k \leq e(|x|)$, $0 \leq j \leq i n_{2}$, and $1 \leq l \leq i$ to the oracle set $B_{4}$. (Note: In this step, $T$ asks the queries of the form $\left\langle x, i, f_{k}, j, l\right\rangle$ for all possible values $i, k, j$, and $l$ because the machine needs to prepare all queries independently of each other.)

Step 6: For each $1 \leq k \leq e(|x|), T$ checks whether $\left\langle x, n_{1}, f_{k}, m_{k}\right\rangle \in B_{3}$. This is done by asking the queries $\left\langle x, i, f_{k}, j\right\rangle$ with $0 \leq i \leq n_{x}, 1 \leq k \leq e(|x|)$, and $0 \leq j \leq i n_{2}$ to the oracle set $B_{3}$. If for some $k,\left\langle x, n_{1}, f_{k}, m_{k}\right\rangle \notin B_{3}$, then $T$ outputs $a_{k, 1} a_{k, 2} \cdots a_{k, n_{1}}$ and halts; otherwise, $T$ outputs the special symbol \# and halts.

Let $F$ denote the function computed by $T$ with oracle $B$. We can easily see that $T$ is polynomial-time bounded and all query strings are prepared independently of each other; this means that the query strings made by $T$ on input $\langle x, w\rangle$ can be realized as parallel queries to the oracle set $B$. Thus, $F$ is in $\mathrm{FP}_{\|}^{\Sigma_{2}^{P}}$.

Let $G$ be a multi-valued function defined by $G(x)=\left\{F(x, w): w \in\{0,1\}^{p(|x|)}\right.$ and $F(x, w)$ is defined $\}-\{\#\}$. We show that $G$ solves $Q$ and is in $R P \cdot \mathrm{FP}_{\|}^{\Sigma_{2}^{\mathrm{P}}}$. To this end, we first prove two claims.

Claim 1 Suppose that for some $k$ with $1 \leq k \leq e(|x|), x$ has a unique solution with 
length $n_{1}$ and of weight $m_{k}$ under $f_{k}$. Then, the string $a_{k, 1} a_{k, 2} \cdots a_{k, n_{1}}$ output by $T$ is a maximal solution of $x$.

Claim 2 Suppose that $x$ has solutions and $w$ is randomly chosen from $\{0,1\}^{p(|x|)}$. Then, $\operatorname{Pr}[F(x, w)$ is a maximal solution of $x] \geq 1-2^{-e(|x|)}$.

Proof. Since $w$ is randomly chosen from $\{0,1\}^{p(|x|)}$, the functions $f_{1}, f_{2}, \cdots, f_{e(|x|)}$ constructed in Step 3 must be random $n_{2}$-weight functions over $\left[1, n_{x}\right]$. Note that $n_{2}=$ $2^{1+\left\lceil\log _{2} n_{x}\right\rceil} \geq 2 n_{x}$. Thus, from Claim 1 and Lemma 3.1, we have that

$\operatorname{Pr}[F(x, w)$ is a maximal solution of $x]$

$=\operatorname{Pr}\left[(\exists k, 1 \leq k \leq e(|x|)) a_{k, 1} a_{k, 2} \cdots a_{k, n_{1}}\right.$ is a maximal solution of $\left.x\right]$

$\geq \operatorname{Pr}[(\exists k, 1 \leq k \leq e(|x|)) x$ has a unique solution with maximum length and of maximum weight under $f_{k}$ ]

$\geq 1-\Pi_{k=1}^{e(|x|)} \operatorname{Pr}[x$ has two or more solutions with maximum length and of maximum weight under $f_{k}$ ]

$\geq 1-\left(\frac{1}{2}\right)^{e(|x|)}=1-2^{-e(|x|)}$.

In the case when $x$ has no solution, $F(x, w)$ is undefined for all $w \in\{0,1\}^{p(|x|)}$ by Step 1 and hence $G(x)=\emptyset$. On the other hand, when $x$ has solutions, $G(x) \neq \emptyset$ by Claim 2 and the definition of $G$, and each element of $G(x)$ is a maximal solution of $x$ by Claim 1. Therefore, $G$ solves $Q$.

If $G(x)=\emptyset$, we know that $x$ has no solution by the discussions in the last paragraph and thus that $F(x, w)$ is undefined for all $w \in\{0,1\}^{p(|x|)}$ by Step 1. On the other hand, if $G(x) \neq \emptyset$, then $x$ has solutions by the discussions in the last paragraph, $\operatorname{Pr}[F(x, w) \in$ $G(x) \cup\{\#\}]=1$ by Step 6 and the definition of $G$, and $\operatorname{Pr}[F(x, w) \in G(x)] \geq 1-2^{-e(|x|)}$ by Claim 2. Therefore, $G$ is in RP.FP ${ }_{\|}^{\Sigma_{2}^{\text {P }}}$.

\section{Hardness of solving MAXP's}

In the light of Proposition 3.1(1), the following proposition shows that FP is a tight lower bound on the complexity of solving MAXP's whose instance-solution relation is P-decidable and hereditary.

Proposition 4.1 There is MAXP $Q=(D, R)$ such that $R$ is P-decidable and hereditary and $Q$ is hard for FP.

By Proposition 3.1(2), the following theorem shows that NPMV //OptP $[O(\log n)]$ is a tight lower bound on the complexity of solving MAXP's whose instance-solution relation is P-decidable but not hereditary.

Theorem 4.1 There is a MAXP $Q=(D, R)$ such that $R$ is P-decidable (but not hereditary) and $Q$ is hard for NPMV //OptP $[O(\log n)]$.

The following corollary is immediate from the proof of Theorem 4.1.

Corollary 4.1 The following problem (called $X$-MaxModel hereafter) is complete for NPMV //OptP $[O(\log n)]$ :

Instance: A CNF boolean formula $\phi$ and a subset $X$ of the set of variables in $\phi$.

Output: A truth assignment $\vec{a}$ to the variables in $X$ such that $\vec{a}$ can be extended to a satisfying truth assignment to $\phi$ but no $\vec{b}$ with $\vec{a} \subset \vec{b}$ and $|\vec{a}|=|\vec{b}|$ can be extended to 
a satisfying truth assignment to $\phi$.

$X$-MaxModel is essentially the same problem as considered by Papadimitriou in Section 3 of [9]. In [9], Papadimitriou claimed without a precise proof that the problem is complete for FP ${ }^{\mathrm{NP}}$. However, he later withdrew his claim and thus left the complexity of the problem open [10]. In [3], we proved that the complexity of the problem is roughly captured by FP $\|$. Now, Corollary 4.1 gives, for the first time, the exact complexity of the problem. Corollary 4.1 is also of special interest in the sense that no natural problem complete for NPMV//OptP $[O(\log n)]$ has been shown before.

By modifying the proof of Theorem 4.1, we can show that NPMV //OptP $[O(\log n)]$ is a tight lower bound on the complexity of solving MAXP's whose instance-solution relation is NP-decidable and hereditary.

Theorem 4.2 There is a MAXP $Q=(D, R)$ such that $R$ is NP-decidable and hereditary and $Q$ is hard for NPMV//OptP $[O(\log n)]$.

The instance-solution relation of $X$-MaxModel is NP-decidable but not hereditary. A natural question arises: Are there natural MAXP's $Q$ such that $Q$ is hard for NPMV/ $/ \mathrm{OptP}[O(\log n)]$ and the instance-solution relation of $Q$ is either NP-decidable and hereditary or P-decidable (but not hereditary)? Unfortunately, we are unable to settle this question. However, we below show that the question will have a positive answer if $\mathrm{NPMV} / / \mathrm{OptP}[O(\log n)]$ in it is replaced by $\mathrm{FP}_{\|}^{\mathrm{NP}}$.

Definition 4.1 A MAXP $Q=(D, R)$ is paddable if there are two functions $f$ and $g$ in FP such that for every list $I=\left\langle x_{1}, x_{2}, \cdots, x_{m}\right\rangle$ of instances of $Q, f(I) \in D$ and for every maximal solution $w$ of $f(I), g(I, w)$ gives a maximal solution for each $x_{i}$.

Lemma 4.1 If a MAXP is paddable and hard for NP, then it is hard for FP $P_{\|}^{N P}$.

Theorem 4.3 The following MAXP's are hard for FP ${ }_{\|}^{N P}$.

(1) MAXIMAL MODEL (MaxModel)

Instance: A CNF boolean formula $\phi$.

Output: A maximal satisfying truth assignment to $\phi$, i.e., a satisfying truth assignment $\vec{a}$ to $\phi$ such that there is no other satisfying truth assignment $\vec{b}$ to $\phi$ with $\vec{a} \subset \vec{b}$.

(2) MAXIMAL CUBIC SUBGRAPH (MaxCubSubgraph)

Instance: An undirected graph $G$.

Output: A maximal subset $F$ of $E(G)$ such that every vertex in the graph $(V(G), F)$ has either degree 3 or degree 0 . (Note: $V(G)$ and $E(G)$ denote hereafter the sets of vertices and edges of $G$, respectively.)

(3) MAXIMAL SATISFIABILITY (MaxSat)

Instance: A CNF boolean formula $\phi=\left\{C_{1}, C_{2}, \cdots, C_{m}\right\}$.

Output: A maximal subset $\phi^{\prime}$ of $\phi$ that is satisfiable.

(4) MAXIMAL $k$-COLORABILITY $(k \geq 3)$ (Max- $k$-Colorability)

Instance: An undirected graph $G$.

Output: A maximal subset of $V(G)$ whose induced subgraph is $k$-colorable.

(5) MAXIMAL HAMILTONIAN SUBGRAPH (MaxHamSubgraph)

Instance: A pair $\langle G, w\rangle$ of a connected undirected graph and a vertex in $G$. 
Output: A maximal subset $U$ of $V(G)$ such that $w \in U$ and the subgraph induced by $U$ has a Hamiltonian circuit.

We here note that a different proof for the $\mathrm{FP}_{\|}^{\mathrm{NP}}$-hardness of MaxModel has been given in [3]. Note that the instance-solution relations of the first two problems in Theorem 4.3 are P-decidable but not hereditary, while the instance-solution relations of the third and fourth problems in Theorem 4.3 are NP-decidable and hereditary. The last problem in Theorem 4.3 is a concrete MAXP whose instance-solution relation is NP-decidable but not hereditary.

For those MAXP's $Q$ whose instance-solution relation is coNP-decidable and hereditary, we are only able to show a loose lower bound.

Proposition 4.2 There is a MAXP $Q=(D, R)$ such that $R$ is a coNP-decidable hereditary relation and $Q$ is hard for $\mathrm{FP}_{\|}^{\mathrm{NP}}$.

In the light of Theorem 3.1(2), the following theorem shows that $\mathrm{FP}_{\|}^{\Sigma_{2}^{\mathrm{P}}}$ is a nearly optimal lower bound on the complexity of solving MAXP's whose instance-solution relation is coNP-decidable but not hereditary.

Theorem 4.4 There is a MAXP $Q=(D, R)$ such that $R$ is coNP-decidable and $Q$ is hard for $\mathrm{FP}_{\|}^{\Sigma_{2}^{\mathrm{P}}}$.

\section{Characterizations of coNP, $\mathbf{D}^{\mathrm{P}}$ and $\Pi_{2}^{\mathrm{P}}$}

The following proposition can be easily proved.

Proposition 5.1 Let $Q=(D, R)$ be a MAXP.

(1) If $R$ is $\mathrm{P}$-decidable and hereditary, then $L_{Q}$ is in $\mathrm{P}$.

(2) If $R$ is $\mathrm{P}$-decidable, then $L_{Q}$ is in coNP.

(3) If $R$ is NP-decidable, then $L_{Q}$ is in $\mathrm{D}^{\mathrm{P}}$.

(4) If $R$ is coNP-decidable and hereditary, then $L_{Q}$ is in $D^{P}$.

(5) If $R$ is coNP-decidable, then $L_{Q}$ is in $\Pi_{2}^{\mathrm{P}}$.

Similar to Proposition 4.1, we can simply show that $\mathrm{P}$ is a tight lower bound on the complexity of $L_{Q}$ for MAXP's $Q$ whose instance-solution relation is P-decidable and hereditary.

The following theorem gives us characterizations of coNP, $D^{P}$, and $\Pi_{2}^{P}$ via MAXP's.

Theorem 5.1 The following hold:

(1) A set $L$ is in coNP if and only if it can be expressed as $L=\{x: f(x)$ is a maximal solution of $x$ in $Q\}$ for some $f \in \mathrm{FP}$ and some MAXP $Q$ whose instance-solution relation is P-decidable.

(2) A set $L$ is in $\mathrm{D}^{\mathbf{P}}$ if and only if it can be expressed as $L=\{x: f(x)$ is a maximal solution of $x$ in $Q\}$ for some $f \in \mathrm{FP}$ and some MAXP $Q$ whose instance-solution relation is NP-decidable (and hereditary).

(3) A set $L$ is in $D^{P}$ if and only if it can be expressed as $L=\{x: f(x)$ is a maximal solution of $x$ in $Q\}$ for some $f \in \mathrm{FP}$ and some MAXP $Q$ whose instance-solution relation is coNP-decidable and hereditary. 
(4) A set $L$ is in $\Pi_{2}^{\mathbf{P}}$ if and only if it can be expressed as $L=\{x: f(x)$ is a maximal solution of $x$ in $Q\}$ for some $f \in \mathrm{FP}$ and some MAXP $Q$ whose instance-solution relation is coNP-decidable.

From the proof of Theorem 5.1(1), we easily see that there is a MAXP whose instancesolution relation is $\mathrm{P}$-decidable (but not hereditary) and whose associated decision problem is $\leq_{m}^{\mathbf{P}}$-complete for coNP. However, the following proposition gives us two concrete such MAXP's.

Proposition 5.2 The decision problems associated with MaxModel and MaxCubSubgraph are $\leq_{m}^{\mathbf{P}}$-complete for coNP:

The following corollary follows immediately from the proof of Theorem 5.1(2) and Cook's theorem.

Corollary 5.1 The decision problem associated with $X$-MaxModel is $\leq_{m}^{\mathbf{P}}$-complete for $D^{P}$.

We next show three natural MAXP's whose instance-solution relations are in NP and whose associated decision problems are $\leq_{m}^{\mathbf{P}}$-complete for $\mathrm{D}^{\mathbf{P}}$.

Proposition 5.3 The decision problems associated with MaxSat, Max- $k$-Colorability and MaxHamSubgraph are $\leq_{m}^{\mathbf{P}}$-complete for $\mathrm{D}^{\mathbf{P}}$ :

We next show a natural MAXP whose instance-solution relation is coNP-decidable and hereditary and whose associated decision problem is $\leq_{m}^{\mathbf{P}}$-complete for $\mathrm{D}^{\mathbf{P}}$. Other such natural MAXP's may be found in $[2,11,12]$.

Proposition 5.4 The following problem is $\leq_{m}^{\mathrm{P}}$-complete for $\mathrm{D}^{\mathrm{P}}$ :

Instance: A triple $\langle\phi, X, \vec{a}\rangle$, where $\phi$ is a CNF boolean formula, $X$ is a set of variables appearing only positively in $\phi$, and $\vec{a}$ is a truth assignment to the variables in $X$.

Question: Is it the case that $\vec{a}$ has no extension satisfying $\phi$ but each $\vec{b} \in \Sigma^{\|X\|}$ with $\vec{a} \subset \vec{b}$ has an extension satisfying $\phi$ ?

\section{Conclusion}

In this paper, we have suggested a general framework for studying the complexity of solving maximization problems. Our results are summarized in Table 1 and Table 2. The results give, systematically, characterizations of several important complexity classes via MAXP's. An important consequence of the results is that the complexity of the problem $X$-MinModel is exactly captured by NPMV//OptP $[O(\log n)]$, giving an answer to an open question of Papadimitriou [9].

As seen from Table 1, the complexity of solving those MAXP's whose instance-solution relation is coNP-decidable and hereditary is unclear. Two obvious open questions are to ask whether the trivial upper bound $\mathrm{FP}^{\mathrm{NP}}$ can be lowered and to ask whether the trivial lower bound $\mathrm{FP}_{\|}^{\mathrm{NP}}$ can be raised. As a step toward the investigation of the two questions, we may first consider what is the complexity of solving MinUnsat (or other natural such problems). Although FP $\|$ is a loose lower bound, proving the FP ${ }_{\|}^{N P}$-hardness of solving MinUnsat seems to be a hard task in the sense that at least the ideas used in proving the 
$\mathrm{D}^{\mathbf{P}}$-hardness of the decision problem associated with MinUnsat do not work [11]. Also, showing that MinUnsat is solvable in a class below FPNP needs new ideas; at least, our ideas used in the proof of Theorem 3.1 do not seem to be applicable.

It would be also interesting to consider the complexity of MAXP's whose instancesolution relation is $\mathbf{C}$-decidable and hereditary for some complexity class $\mathbf{C}$ below $\mathbf{P}$. These MAXP's are obviously solvable in FP. Are they solvable in a class below FP or is there such a MAXP $Q$ that solving $Q$ is complete for FP (say, under $\leq_{1-\mathrm{T}}^{\mathrm{NC}}$ reductions)? The two questions are important in parallel computation in the case when $\mathbf{C} \subseteq$ NC.

\section{References}

[1] R. Anderson, A Parallel Algorithm for the Maximal Path Problem, Proc. 17th ACM Symposium on Theory of Computing (1985) 33-37.

[2] J.-Y. Cai and G.E. Meyer, Graph Minimal Uncolorability is $\mathrm{D}^{\mathrm{P}}$-Complete, SIAM J. Comput., 16 (1987), pp. 259-277.

[3] Z.-Z. Chen and S. Toda, The Complexity of Finding a Minimal Satisfying Truth Assignment to a Boolean Formula, In submission (1992).

[4] Z.-Z. Chen, A Randomized NC Algorithm for the Maximal Tree Cover Problem, Inform. Process. Lett. 40 (1991) 241-246.

[5] R.M. Karp and A. Wigderson, A Fast Parallel Algorithm for the Maximal Independent Set Problem, Proc. 16th ACM Symposium on Theory of Computing (1984) 266-272.

[6] M.W. Krentel, The Complexity of Optimization Problems, J. Comput. System Sci., 36 (1988), pp. 490-509.

[7] M. Luby, A Simple Parallel Algorithm for the Maximal Independent Set Problem, Proc. 17th ACM Symposium on Theory of Computing (1985) 1-10.

[8] K. Mulmuley, U.V. Vazirani and V.V. Vazirani, Matching is as Easy as Matrix Inversion, Proc. 19th ACM Symposium on Theory of Computing (1987), pp. 345354.

[9] C.H. Papadimitriou, On Selecting a Satisfying Truth Assignment, Proc. 32nd IEEE Symposium on the Foundations of Computer Science (1991), pp. 163-169.

[10] C.H. Papadimitriou, Personal Communication (1992).

[11] C.H. Papadimitriou and D. Wolfe, The Complexity of Facets Resolved,, Proc. 26nd IEEE Symposium on the Foundations of Computer Science (1985), pp. 74-78.

[12] C.H. Papadimitriou and M. Yannakakis, The Complexity of Facets (and Some Facets of Complexity), J. Comput. System Sci., 28 (1982), pp. 244-259. 